Кииническое наблюдение / Case report

ISSN 1995-5464 (Print); ISSN 2408-9524 (Online)

https://doi.org/10.16931/1995-5464.2021-4-126-132

Симультанная трансоральная

паратиреоиАэктомия и ^апароскопическая

околоопухолевая резекшия поАкелудочной

железы при синдроме множественной

энАокринной неоплазии 1 типа

Грязнов С.Е. ${ }^{\text {* }, ~ Б у р и е в ~ И . М . ~}{ }^{1}$, Мелконян Г.Г. ${ }^{1,2}$, Малюга Н.С. ${ }^{1}$, Лайпанов Б.К. ${ }^{1}$

${ }^{1}$ ГБУз “Городская клиническая больница №4 “Павловская” Департамента здравоохранения города Москвы”; 115093, Москва, ул. Павловская, д. 25, Российская Федерация

2 ФГБОУ ДПО “Российская медицинская академия непрерывного профессионального образования"

Министерства здравоохранения РФ; 125993, Москва, ул. Павловская, д. 2/1, стр. 1,

Российская Федерация

Представлено клиническое наблюдение пациентки с синдромом множественной эндокринной неоплазии 1 типа (МЭН 1). Диагностировано сочетание первичного гиперпаратиреоза, аденомы околощитовидной железы и гормонально неактивной опухоли головки поджелудочной железы. Проведено хирургическое лечение симультанная трансоральная паратиреоидэктомия и лапароскопическая резекция головки поджелудочной железы. Описание таких вмешательств в рамках одной операции у пациента с синдромом МЭН 1 не найдено. Применено оригинальное решение для выполнения интраоперационного ультразвукового исследования. Представлены результаты динамического наблюдения в течение года после операции. Продемонстрированы возможности эндоскопических технологий при синдроме МЭН 1.

Ключевые слова: множественная эндокринная неоллазия, околощитовидная железа, гиперпаратиреоз, поджелудочная железа, нейроэндокринная опухоль, трансоральная паратиреоидэктомия, интраоперационное УзИ, симультанная операция

Ссылка для цитирования: Грязнов С.Е., Буриев И.М., Мелконян Г.Г., Малюга Н.С., Лайпанов Б.К. Симультанная трансоральная паратиреоидэктомия и лапароскопическая околоопухолевая резекция поджелудочной железы при синдроме множественной эндокринной неоплазии 1 типа. Анналы хирургической гепатологии. 2021; 26 (4): $126-132$. https://doi.org/10.16931/1995-5464.2021-4-126-132.

Авторы подтверждают отсутствие конфликтов интересов.

\title{
Simultaneous transoral parathyroidectomy and laparoscopic pancreatic resection in type 1 Multiple Endocrine Neoplasia syndrome
}

Gryaznov S.E. ${ }^{\text {* }}$, Buriev I.M. ${ }^{1}$, Melkonyan G.G. ${ }^{1,2}$, Malyuga N.S. ${ }^{1}$, Laypanov B.K. ${ }^{1}$

${ }^{1}$ City Clinical Hospital No.4, Moscow Healthcare Department; 25, Pavlovskaia str., Moscow, 115093,

Russian Federation

${ }^{2}$ Russian Medical Academy of Continuous Professional Education of the Ministry of Health of Russia; bld. 1, 2/1, Barricadnaya str., Moscow, 125993, Russian Federation

The article presents a clinical observation of a patient with type 1 Multiple Endocrine Neoplasia syndrome (MEN 1). During the diagnostic search, a combination of primary hyperparathyroidism, parathyroid adenoma and hormonally inactive pancreatic head tumor was found. Simultaneous transoral parathyroidectomy and laparoscopic resection of the pancreatic head was performed. We haven`t found the literature data describing such kind of operations for MEN 1 syndrome. An original solution was applied to perform intraoperative ultrasonography monitoring. The results of 1-year postoperative follow-up are presented. This observation demonstrates the possibilities of endoscopic technologies in the treatment of MEN 1 syndrome.

Keywords: multiple endocrine neoplasia, MEN 1, parathyroid gland, hyperparathyroidism, neuroendocrine tumor, pancreas, transoral parathyroidectomy, intraoperative ultrasonography, simultaneous surgery 
For citation: Gryaznov S.E., Buriev I.M., Melkonyan G.G., Malyuga N.S., Laypanov B.K. Simultaneous transoral parathyroidectomy and laparoscopic pancreatic resection in type 1 Multiple Endocrine Neoplasia syndrome. Annaly khirurgicheskoy gepatologii = Annals of HPB surgery. 2021; 26 (4): 126-132. (In Russian).

https://doi.org/10.16931/1995-5464.2021-4-126-132.

There is no conflict of interests.

Синдром множественной эндокринной неоплазии 1 типа (МЭН 1), или синдром Вермера, это генетически детерминированное заболевание с аутосомно-доминантным типом наследования. В $10 \%$ наблюдений выявляют спорадические наблюдения в результате мутации de поvо. Синдром включает различные комбинации опухолей эндокринных желез, но чаще всего характеризуется поражением околощитовидных желез (ОЩЖ), гипофиза и поджелудочной железы (ПЖ) [1]. Диагноз устанавливают при обнаружении опухолей как минимум в двух из трех органов. Примерно у 95\% пациентов с МЭН 1 первым клиническим проявлением синдрома является первичный гиперпаратиреоз (ПГПТ) с гиперплазией или аденомой в одной или, чаще, в нескольких ОЩЖ [1]. Опухоль ПЖ выявляют у 40-70\% пациентов с МЭН 1. Наиболее часто это нефункционирующая опухоль $(80-100 \%)$, гастринома (20-61\%), инсулинома (7-31\%) [2]. В 30-40\% наблюдений синдрома МЭН 1 выявляют новообразование передней доли гипофиза - пролактиному (60\%) и опухоли, секретирующие гормон роста (25\%) [3].

Лечение пациентов с синдромом МЭН 1 в целом аналогично лечению при изолированных опухолях соответствующих органов. Однако результаты его при МЭН 1 не такие успешные. Это связано в первую очередь с множественностью опухолевых очагов и большим риском рецидива [4]. При ПГПТ в рамках МЭН 1 общепризнанными являются операции в объеме субтотальной паратиреоидэктомии или паратиреоидэктомии с аутотрансплантацией [4]. В ряде исследований показано, что при тщательной дооперационной локализации патологически измененных ОЩЖ у некоторых пациентов можно уменьшить объем операции вплоть до удаления солитарной аденомы. Рецидив заболевания при продолжительном динамическом наблюдении не развивался $[5,6]$.

Целью хирургического лечения при нейроэндокринных опухолях (НЭО) ПЖ является уменьшение риска рецидива и метастазирования опухоли, а также более длительное сохранение экзокринной и эндокринной функции железы [7]. Согласно международным рекомендациям, критерием хирургического лечения функционально активных НЭО является размер опухоли $>1$ см [8]. При нефункционирующих опухолях до 2 см предложена наблюдательная тактика [9]. С другой стороны, при опухолях $>1$ см рекомендуют хирургическое лечение [10].
Лечение опухолей гипофиза включает как медикаментозную терапию, так и хирургическое вмешательство; методом выбора является эндоскопическое удаление опухоли [11].

Очередность хирургического лечения при синдроме МЭН 1 типа с поражением двух или более органов-мишеней следует определять индивидуально, в зависимости от тяжести заболевания и прогноза. В большинстве наблюдений этапы хирургического лечения разнесены во времени, однако опубликованы сообщения и о симультанных вмешательствах $[12,13]$. Приводим клиническое наблюдение.

Пациентка 54 лет, проживает в Сочи, обратилась за консультацией в ГКБ №4 в январе 2020 г. в связи с выявленным образованием в головке ПЖ и подозрением на ПГПТ. Считает себя больной с августа 2019 г., когда стали беспокоить эпизоды сердцебиения, повышения АД, “ломота” в костях, боль в суставах, общее плохое самочувствие. Связывает начало заболевания с сильным эмоциональным потрясением смертью родителей (у матери - холангиокарцинома с метастазами в печень и легкие). С указанными жалобамипациентка обратиласьктерапевту.Диагностирована гипертоническая болезнь, назначено обследование. По данным УЗИ в головке ПЖ выявлено образование $10 \times 11$ мм. Выполнена КТ органов брюшной полости с внутривенным контрастным усилением. В области головки ПЖ - опухоль $10 \times 11$ мм 32-44 HU. Выявлены признаки слабоинтенсивного контрастного усиления, преимущественно по периферии в артериальную фазу, хорошо выраженное равномерное контрастное усиление в венозную и отсроченную фазы до 134-167 HU. Учитывая жалобы и наблюдение эндокринологом в анамнезе по поводу мелких узловых образований в ЩЖ, проведено лабораторное исследование гормонов ЩЖ и электролитов крови. Тиреотропный гормон и $\mathrm{T}_{4}$ были в пределах допустимых значений. Общий кальций 3,07 ммоль/л (2,10-2,55), паратгормон 210,5 пг/мл (15-65). Учитывая гиперкальциемию и повышение уровня паратгормона, заподозрен ПГПТ, рекомендована сцинтиграфия ОЩЖ. Для дальнейшего обследования пациентка обратилась в ГКБ № 4 г. Москвы. При первичной консультации заподозрен синдром МЭН 1. Повторное исследование общего Са крови подтвердило его повышение до 2,71 ммоль/л $(2,1-2,55)$. Выявлено повышение ионизированного кальция крови до 1,47 ммоль/л $(1,05-1,35)$ и снижение витамина D до 22,4 нг/мл (10-30 нг/мл - недостаток). Выполнено УЗИ. В проекции нижней ОЩЖ справа выявлена опухоль $2,56 \times 1,68$ см. Сцинтиграфия 
с однофотонной эмиссионной КТ: накопление радиофармпрепарата по заднему контуру правой доли ЩЖ на уровне ее нижней трети. По данным 3D-peконструкции дополнительных очагов накопления не выявлено, диагностирована аденома правой нижней ОЩЖ. В почках при УЗИ патологических изменений не выявлено. ЭГДС: очаговый гастрит, недостаточность кардии. Двухэнергетическая рентгеновская денситометрия в режиме аксиального сканирования: снижение минеральной плотности L1-L3. T-критерий $-2,19 \mathrm{SD}$, что соответствует остеопении 3-й степени. При УЗИ и КТ подтверждено новообразование в головке ПЖ. Изменений в надпочечниках не выявлено. МРТ головного мозга с контрастным усилением - патологических изменений в гипофизе нет. Инсулин, гастрин, пролактин, хромогранин А в пределах допустимых значений. Онкомаркеры СА 15-3, СА 125, СА 19-9, SCСА, РЭА, кальцитонин также в пределах нормы. Рекомендовано генетическое исследование мутации в гене $M E N 1$, однако пациентка от него воздержалась. В результате обследования диагностирован синдром МЭН 1, ПГПТ, мягкая форма, аденома правой нижней ОЩЖ, гормонально неактивная опухоль головки ПЖ. 13.02.2020 пациентка оперирована. Первым этапом выполнена трансоральная паратиреоидэктомия (рис. 1). Доступ в нижнем своде преддверия рта с трехточечной расстановкой инструментов центральный 11-мм троакар для 5-мм видеоэндоскопа, два 5-мм троакара для рабочих инструментов билатерально на уровне первых премоляров. Поддержание рабочей полости инсуффляцией газа с давлением 6 мм рт.ст. При ревизии по заднебоковой поверхности правой доли ЩЖ на уровне ее нижней трети выявлена аденома ОЩЖ. Опухоль удалена с помощью ультразвуковых ножниц. Препарат эвакуирован в контейнере через центральный 11-мм порт. Продолжительность первого этапа составила 125 мин, кровопотеря - 10 мл. Дренирование не выполняли. Вторым этапом выполнена лапароскопическая околоопухолевая резекция головки ПЖ (рис. 2). При об- зорной лапароскопии патологических изменений не выявлено. После рассечения желудочно-ободочной связки обнажена передняя поверхность головки ПЖ, поверхность ее не изменена. Через дополнительный 3-см разрез в эпигастрии в толщу круглой связки заведен ультразвуковой датчик (мультичастотный микроконвексный внутрипросветный, 2,1-4,4 МГц) в стерильном латексном рукаве, и выполнено интраоперационное УЗИ. В паренхиме головки ПЖ выявлено образование $1 \times 1$ см (рис. 3). Проекция образования на поверхность железы маркирована коагуляцией. Далее выполнена резекция опухоли в пределах здоровых тканей с использованием биполярной коагуляции и ультразвуковых ножниц. Гемостаз, дренаж к головке ПЖ и ушивание ран. Продолжительность второго этапа составила 185 мин, кровопотеря 90 мл. Таким образом, была выполнена симультанная последовательная трансоральная эндоскопическая паратиреоидэктомия справа, лапароскопическая околоопухолевая резекция головки ПЖ. Общее время нахождения пациентки в наркозе составило 395 мин. В послеоперационном периоде, как и ожидали, по причине коагуляционного некроза и травмы ацинусов у пациентки сформировался панкреатический свищ, который закрылся через 2 мес. По данным УЗИ на 2-е сутки после операции - следы свободной жидкости в брюшной полости. На 5-е сутки при УЗИ в брюшной полости патологических изменений не выявлено. Выписана на 7-е сутки после операции в удовлетворительном состоянии с дренажом брюшной полости, по которому выделялось до 100 мл в сутки. Рекомендован прием креона 10000 Ед 3 раза в сутки, лабораторный мониторинг. На 10-е сутки после операции лейкоциты крови $6,85 \times 10^{9} /$ л $(4,5-$ $11)$, гемоглобин 14 г/дл (11,7-16), амилаза крови 158 Ед/л (25-125), общий Са 2,26 ммоль/л $(2,1-2,55)$, паратгормон 6,82 пмоль/л (1,6-6,9). В течение месяца после операции сохранялось выделение 100-125 мл по дренажу. На 2-м месяце количество отделяемого постепенно уменьшалось и через 55 дней после опе-
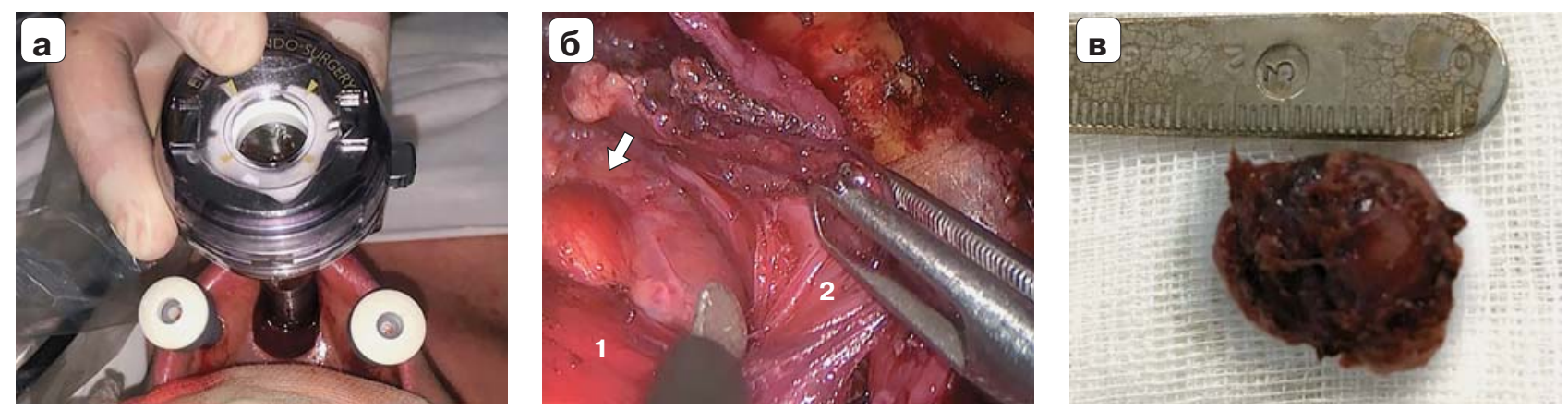

Рис. 1. Трансоральная паратиреоидэктомия: $\mathbf{a}$ - интраоперационное фото, расположение троакаров; $\boldsymbol{\sigma}$ - интраоперационное фото, мобилизация опухоли ОЩЖ; в - макрофото. 1 - правая доля ЩЖ, 2 - правые претиреоидные мышцы. Аденома ОЩЖ указана белой стрелкой

Fig. 1. Transoral parathyroidectomy: a - intraoperative photo of the trocars positioning; $\boldsymbol{\sigma}$ - intraoperative photo of mobilization of the PTG tumor $(1$ - the right lobe of the thyroid gland, 2 - the right prethyroid muscles, the PTG adenoma is indicated with the white arrow); в - macrophoto. 

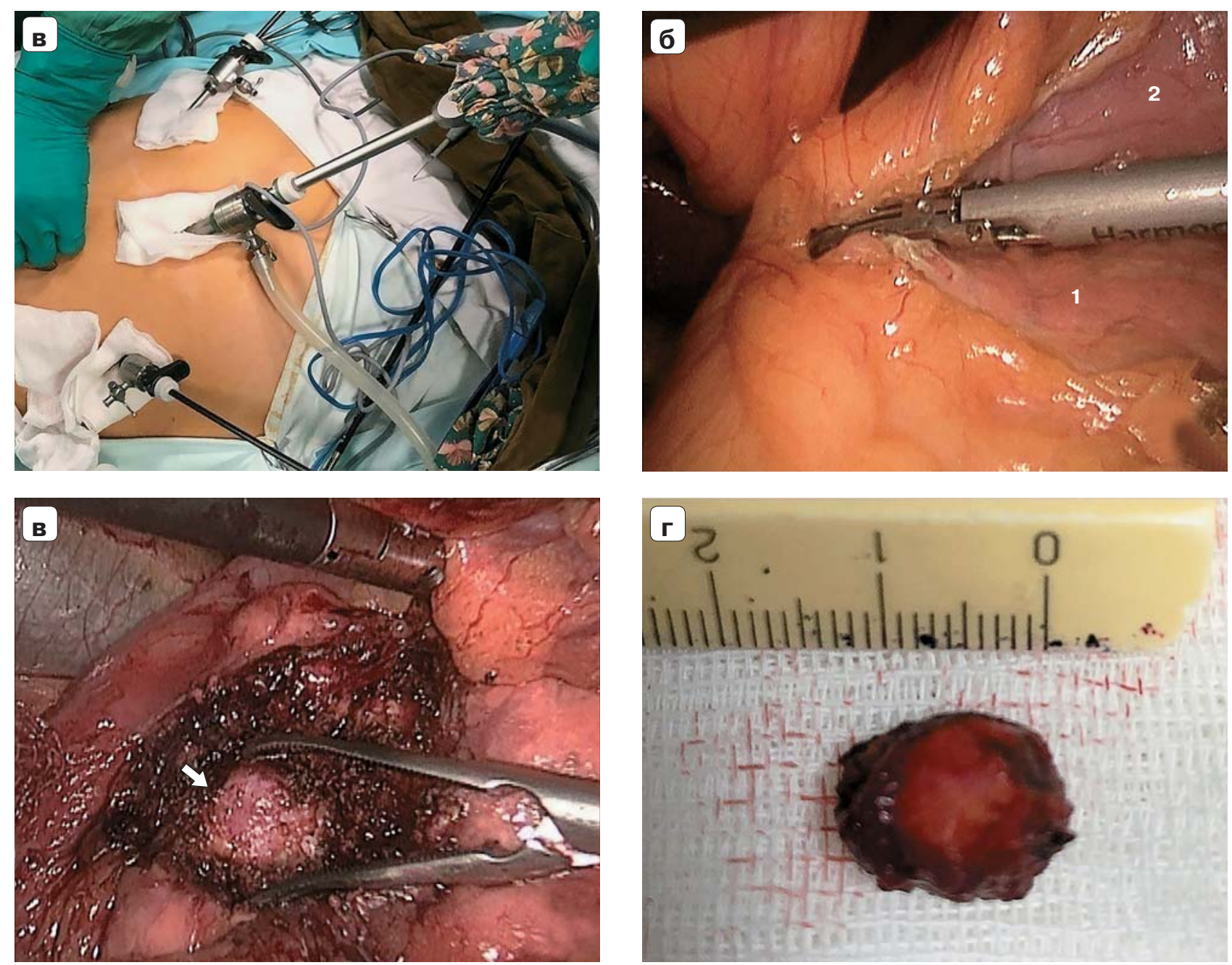

Рис. 2. Лапароскопическая резекция головки ПЖ: а - интраоперационное фото, расположение троакаров; $\boldsymbol{6}$ - интраоперационное фото, этап рассечения желудочно-ободочной связки; в - интраоперационное фото, опухоль в головке ПЖ (указана белой стрелкой); г - макрофото. 1 - ПЖ, 2 - желудок.

Fig. 2. Laparoscopic resection of the pancreatic head: $\mathbf{a}$ - intraoperative photo of the trocars positioning; $\boldsymbol{\sigma}-$ intraoperative photo, the dissection of the gastrocolic ligament (1 - pancreas, 2 - stomach); в - intraoperative photo of a tumor in the head of the pancreas (white arrow); $\mathbf{r}-$ macrophoto.
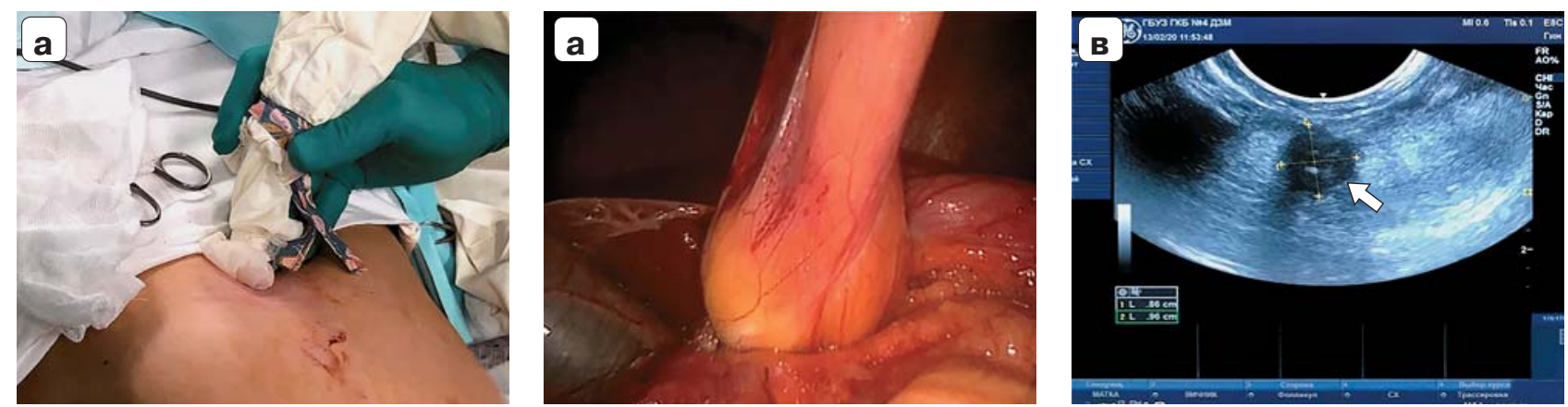

Рис. 3. Интраоперационное УЗИ: а - датчик заведен в брюшную полость через разрез в эпигастрии; б - датчик в круглой связке; в - опухоль в головке ПЖ (указана стрелкой).

Fig. 3. Intraoperative US: $\mathbf{a}-$ the sensor is inserted into the abdominal cavity through an incision in the epigastrium; $\boldsymbol{\sigma}-$ a sensor in the round ligament; в - a tumor in the head of the pancreas (arrow). 

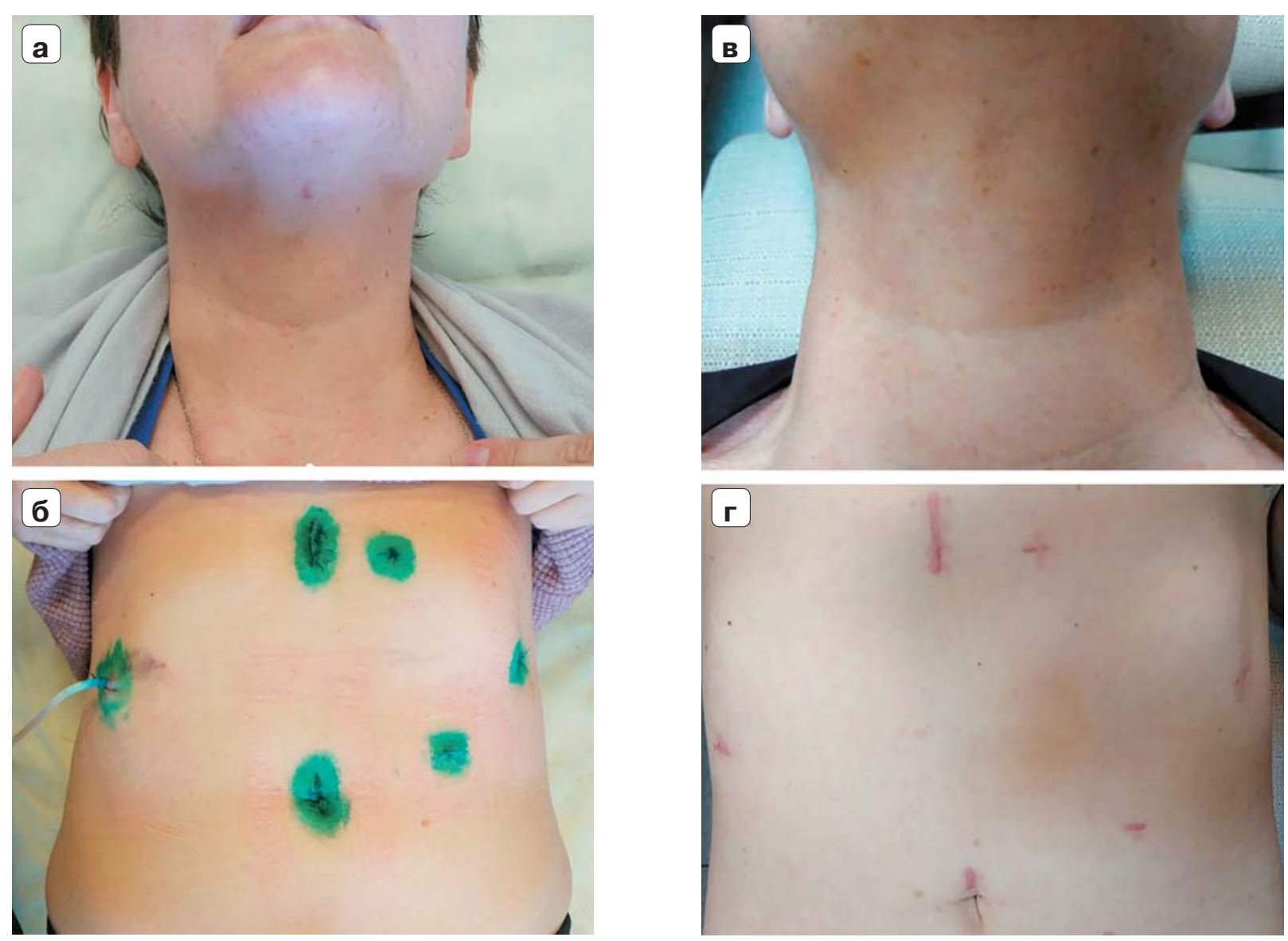

7-е сутки

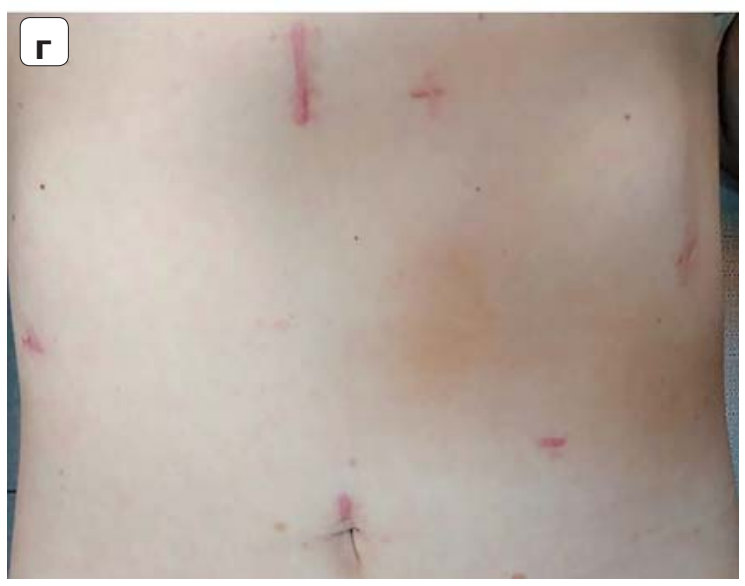

4 мес

Рис. 4. Местный статус: а, $\boldsymbol{\sigma}$ - 7-е сутки после операции; в, г - через 4 мес после операции.

Fig. 4. Local status: a, $\mathbf{\sigma}-$ a week after the operation; в, $\mathbf{r}-4$ month after the operation.
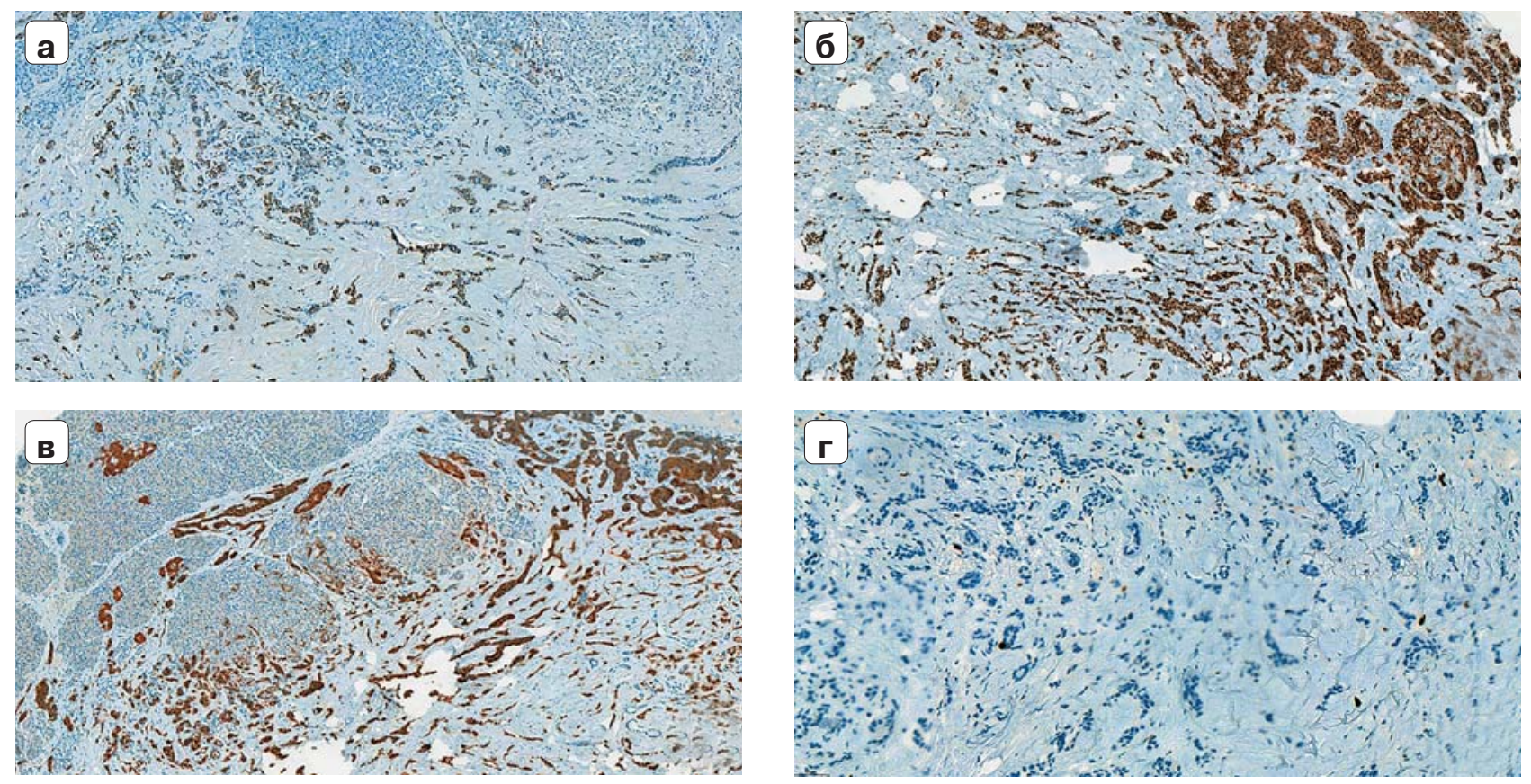

Рис. 5. Микрофото. Иммуногистохимическое исследование: $\mathbf{a}$ - экспрессия синаптофизина; б - экспрессия рецепторов прогестерона; в - экспрессия хромогранина А; г - индекс пролиферации Ki-67 <3\%, G1, митозы отсутствуют.

Fig. 5. Microphoto. Immunohistochemistry: a - synaptophysin expression; $\mathbf{6}$ - expression of progesterone receptors; в expression of chromogranin A; $\mathbf{r}-\mathrm{Ki} 67<3 \%$, G1, mitoses are absent. 
рации дренаж был удален (рис. 4). Гистологическое исследование: светлоклеточная аденома околощитовидной железы. Иммуногистохимическое исследование опухоли ПЖ: опухолевые клетки диффузно экспрессируют синаптофизин, рецепторы прогестерона и хромогранин А. Индекс пролиферации с Кi-67<3\% опухолевых клеток. Митозы отсуствуют. Экспрессия с p53 отрицательная. Заключение: хорошо дифференцированная кистозная НЭО ПЖ G1 (рис. 5). Период наблюдения составил 1 год. Через год общий кальций, ионизированный кальций, паратгормон - в пределах допустимых значений, амилаза крови - 66,2 Ед/л (13-55). При УЗИ ОЩЖ не увеличены. КТ брюшной полости с контрастированием: послеоперационные изменения в головке ПЖ. Также через год после операции проведена оценка качества жизни с использованием опросника SF-36. Общий физический компонент здоровья составил 56,93 балла, общий психический компонент здоровья - 60,46 балла. Результаты свидетельствуют о высоком качестве жизни пациентки через год после оперативного лечения.

Диагностический поиск в отсутствие ярких проявлений заболевания и подтвержденного семейного анамнеза по синдрому МЭН 1 был продуктивным. Это спорадическое наблюдение с поздней манифестацией и практически бессимптомным течением. Безусловно, сочетание ПГПТ и новообразований в области ПЖ является поводом для диагностики синдрома МЭН 1. Для полноты картины необходимо генетическое исследование, однако в представленном наблюдении выполнить его не удалось. После операции психоэмоциональное состояние пациентки значительно улучшилось. Динамическое наблюдение в течение года после операции свидетельствует об отсутствии рецидива. Одним из факторов, оказавших положительное влияние на качество жизни пациентки, стал выбор в пользу мини-инвазивных эндоскопических доступов к органам-мишеням.

Сочетание трансоральной паратиреоидэктомии и лапароскопического вмешательства на ПЖ в рамках симультанной операции при синдроме МЭН 1 выполнено впервые; поиск публикаций в PubMed и eLibrary осуществляли по ключевым словам. Такой подход был обоснован адекватной предоперационной топической диагностикой и возможностью выполнения интраоперационного УЗИ. В связи с отсутствием специального лапароскопического датчика для интраоперационного УЗИ было найдено оригинальное решение - применили микроконвексный полостной датчик, позволивший четко определить локализацию опухоли (метод проходит процедуру получения патента).

На современном этапе развития хирургии симультанные операции при синдроме МЭН 1 оправданы у ряда пациентов, а эндоскопические вмешательства при этом могут стать вариантом выбора. Наблюдение за пациенткой будет продолжено, включая лабораторные исследования, КТ брюшной полости, наблюдение за гипофизом. Также было рекомендовано генетическое обследование близких родственников.

\section{Участие авторов}

Грязнов С.Е. - основной автор статьи, оперирующий хирург, лечащий врач пациентки.

Буриев И.М. - научный консультант исследования, оперирующий хирург, корректор текста.

Мелконян Г.Г. - руководитель исследования, корректор текста.

Малюга Н.С. - выполнение УЗИ и интерпретация результатов.

Лайпанов Б.К. - оперирующий хирург, анализ данных.

\section{Authors participation}

Gryaznov S.E. - main author of the article, operating surgeon, attending physician of the patient.

Buriev I.M. - research consultant, operating surgeon, text proofreader.

Melkonyan G.G. - research leader, text proofreader.

Malyuga N.S. - sonographer, performing ultrasound and interpreting the results.

Laipanov B.K. - operating surgeon, data analysis.

\section{Список литературы [References]}

1. Giusti F., Marini F., Brandi M.L. Multiple Endocrine Neoplasia Type 1. 2005 Aug 31 [updated 2017 Dec 14]. In: Adam M.P., Ardinger H.H., Pagon R.A., Wallace S.E., Bean L.J.H., Stephens K., Amemiya A., editors. GeneReviews ${ }^{\circledR}$ [Internet]. Seattle (WA): University of Washington, Seattle; 1993-2020. PMID: 20301710 .

2. Кригер А.Г., Кочатков А.В., Горин Д.С., Лебедева А.Н. Эндокринные опухоли поджелудочной железы при синдроме множественной эндокринной неоплазии I типа. Хирургия. Журнал им. Н.И. Пирогова. 2010; (8): 69-75.

Kriger A.G., Kochatkov A.V., Gorin D.S., Lebedeva A.N. Endocrine pancreatic tumors by type I syndrome of multiple endocrine neoplasia. Pirogov Russian Journal of Surgery = Khirurgiya. Zhurnal imeni N.I. Pirogova. 2010; (8): 69-75. (In Russian)

3. Vergès B., Boureille F., Goudet P., Murat A., Beckers A., Sassolas G., Cougard P., Chambe B., Montvernay C., Calender A. Pituitary disease in MEN type 1 (MEN1): data from the France-Belgium MEN1 multicenter study. J. Clin. Endocrinol. Metab. 2002; 87 (2): 457-465.

https://doi.org/10.1210/jcem.87.2.8145

4. Schreinemakers J.M., Pieterman C.R., Scholten A., Vriens M.R., Valk G.D., Rinkes I.H. The optimal surgical treatment for primary hyperparathyroidism in MEN1 patients: a systematic review. World J. Surg. 2011; 35 (9): 1993-2005.

https://doi.org/10.1007/s00268-011-1068-9

5. Manoharan J., Albers M.B., Bollmann C., Maurer E., Mintziras I., Wächter S., Bartsch D.K. Single gland excision for MEN1-associated primary hyperparathyroidism. Clin. Endocrinol. (Oxf.). 2020; 92 (1): 63-70. https://doi.org/10.1111/cen.14112 
6. Horiuchi K., Sakurai M., Haniu K., Nagai E., Tokumitsu H., Yoshida Y., Omi Y., Sakamoto A., Okamoto T. Impact of "tailored" parathyroidectomy for treatment of primary hyperparathyroidism in patients with multiple endocrine neoplasia type 1. World J. Surg. 2018; 42 (6): 1772-1778. https://doi.org/10.1007/s00268-017-4366-Z

7. Kiernan C.M., Grubbs E.G. Surgical management of multiple endocrine neoplasia 1 and multiple endocrine neoplasia 2. Surg. Clin. North Am. 2019; 99 (4): 693-709. https://doi.org/10.1016/j.suc.2019.04.015

8. Jensen R.T., Norton J.A. Treatment of pancreatic neuroendocrine tumors in multiple endocrine neoplasia type 1: some clarity but continued controversy. Pancreas. 2017; 46 (5): 589-594. https:// doi.org/10.1097/MPA.0000000000000825

9. Falconi M., Eriksson B., Kaltsas G., Bartsch D.K., Capdevila J., Caplin M., Kos-Kudla B., Kwekkeboom D., Rindi G., Klöppel G., Reed N., Kianmanesh R., Jensen R.T. ENETS Consensus Guidelines Update for the management of patients with functional pancreatic neuroendocrine tumors and non-functional pancreatic neuroendocrine tumors. Neuroendocrinology. 2016; 103 (2): 153-171. https://doi.org/10.1159/000443171

10. Yang G., Ji M., Chen J., Chen R., Chen Y., Fu D., Hou B., Huang H., Jiang L., Jin K., Ke N., Li Y., Li Y., Liang H., Liu A., Luo J., Ni Q., Shao C., Shen B., Sheng W., Song B., Sun J., Tan C., Tan H., Tang Q., Tang Y., Tian X., Wang J., Wang J., Wang W., Wang W., Wu Z., Xu J., Yan Q., Yang N., Yang Y.,
Yin X., Yu X., Yuan C., Zeng S., Zhang G., Zhang R., Zhou Z., Zhu Z., Shao C. Surgery management for sporadic small $(\leqslant 2 \mathrm{~cm})$, non-functioning pancreatic neuroendocrine tumors: a consensus statement by the Chinese Study Group for Neuroendocrine Tumors (CSNET). Int. J. Oncol. 2017; 50 (2): 567-574. https://doi.org/10.3892/ijo.2016.3826

11. Concolino P., Costella A., Capoluongo E. Multiple endocrine neoplasia type 1 (MEN1): An update of 208 new germline variants reported in the last nine years. Cancer Genet. 2016; 209 (1-2): 36-41. https://doi.org/10.1016/j.cancergen.2015.12.002

12. Игнатюк В.Г., Бритвин Т.А., Подрез Д.В., Гуревич Л.Е. Синдром множественной эндокринной неоплазии 1 типа с гиперпаратиреозом, инсулиномой поджелудочной железы и гастриномой ворот печени. Анналы хирургической гепатологии. 2018; 23 (3): 57-63. https://doi.org/10.16931/19955464.2018357-63

Ignatyuk V.G., Britvin T.A., Podrez D.V., Gurevich L.E. Multiple endocrine neoplasia type 1 with primary hyperparathyroidism, pancreatic insulinoma and portal gastrinoma (case report). Annaly khirurgicheskoy gepatologii $=$ Annals of HPB Surgery. 2018; 23 (3): 57-63. https://doi.org/10.16931/19955464.2018357-63 (In Russian)

13. Hou B., Tang W., Su X., Liu W. [Successful simultaneous surgery for patient with insulinoma and parathyroid adenoma relevant to multiple endocrine neoplasia type 1: A case report]. Zhong Nan Da Xue Xue Bao Yi Xue Ban. 2019; 44 (9): 1083-1088. Chinese. https://doi.org/10.11817/j.issn.1672-7347.2019.190601

\section{Сведения об авторах [Authors info]}

Грязнов Сергей Евгеньевич - врач-хирург, онколог ГБУЗ “ГКБ №4 ДЗМ”. https://orcid.org/0000-0003-2074-4130. E-mail: gryzli_37@mail.ru

Буриев Илья Михайлович - доктор мед. наук, профессор, врач-хирург, онколог, советник главного врача по хирургии ГБУЗ “ГКБ №4 ДЗМ”. https://orcid.org/0000-0002-1205-9152. E-mail: imburiev@gmail.com

Мелконян Георгий Геннадьевич - доктор мед. наук, профессор кафедры хирургии ФГБОУ ДПО РМАНПО Минздрава России; главный врач ГБУЗ “ГКБ №4 ДЗМ”. https://orcid.org/0000-0001-7234-4185. E-mail: glav@gkb4.ru

Малюга Наталья Сергеевна - заведующая отделением ультразвуковой диагностики ГБУз “ГКБ №4 ДЗМ”. https://orcid.org/0000-0003-0559-1308. E-mail: maluga@mail.ru

Лайпанов Борис Камалович - канд. мед. наук, врач-хирург ГБУз “ГКБ №4 ДЗМ”. https://orcid.org/0000-0002-5776-3653. E-mail: blaypanov@mail.ru

Для корреспонденции *: Грязнов Сергей Евгеньевич - 115093, Москва, ул. Павловская, д. 25, Российская Федерация. Тел.: 8-916-299-26-88. E-mail: gryzli_37@mail.ru

Sergey E. Gryaznov - Surgeon, Oncologist, City Clinical Hospital No. 4 of the Moscow Healthcare Department. https://orcid. org/0000-0003-2074-4130. E-mail: gryzli_37@mail.ru

Ilya M. Buriev - Doct. of Sci. (Med.), Professor, Surgeon, Oncologist, City Clinical Hospital No. 4 of the Moscow Healthcare Department. https://orcid.org/0000-0002-1205-9152. E-mail: imburiev@gmail.com

Georgy G. Melkonyan - Doct. of Sci. (Med.), Professor of the Department of Surgery, Russian Medical Academy of Continuous Professional Education of the Ministry of Health of Russia; Chief Physician of the City Clinical Hospital No. 4 of the Moscow Healthcare Department. https://orcid.org/0000-0001-7234-4185. E-mail: glav@gkb4.ru

Natalya S. Malyuga - Head of the Department of Ultrasound Diagnostics, City Clinical Hospital No. 4, Moscow Healthcare Department. https://orcid.org/0000-0003-0559-1308. E-mail: maluga@mail.ru

Boris K. Laipanov - Cand. of Sci. (Med.), Surgeon, City Clinical Hospital No. 4 of the Moscow Healthcare Department. https://orcid.org/0000-0002-5776-3653. E-mail: blaypanov@mail.ru

For correspondence*: Sergey E. Gryaznov - 25, Pavlovskaya str., the Moscow Region, 115093, Russian Federation. Phone: 8-916-299-26-88. E-mail: gryzli_37@mail.ru

Статья поступила в редакцию журнала 01.03.2021.

Received 01 March 2021.
Принята к публикации 28.09.2021

Accepted for publication 28 September 2021 